\title{
Factores de riesgo y detección temprana del melanoma maligno en pacientes con nevus melanocíticos
}

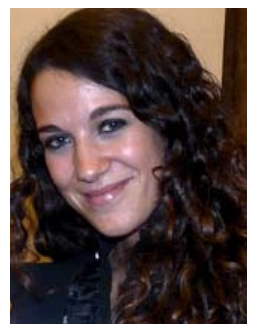

Mar Burgués Calderón Médico residente. Servicio de Dermatología. Hospital Universitario 12 de Octubre. Madrid.

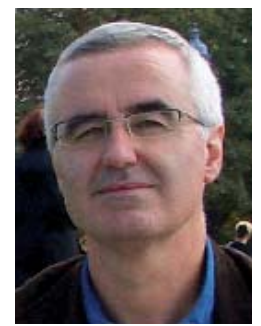

Rafael Llamas Martín Médico adjunto. Servicio de Dermatología. Hospital Universitario 12 de Octubre. Madrid. Profesor asociado de Dermatología. Universidad Complutense de Madrid.

\section{RESUMEN}

El melanoma maligno es un tipo de cáncer de piel que se caracteriza por su gran agresividad y resistencia al tratamiento. Los principales factores de riesgo que se asocian al desarrollo de melanoma maligno son la exposición solar y a otras fuentes de radiación ultravioleta, el fenotipo (pelo, ojos y piel claros), la presencia de antecedentes familiares de melanoma y el síndrome del nevus displásico. El aumento de la incidencia de melanoma maligno en las últimas décadas ha generado un problema de salud pública y ha promovido el desarrollo de técnicas de detección temprana de melanoma. A pesar de que el diagnóstico definitivo es histológico, estas técnicas no invasivas nos pueden ayudar a obtener un diagnóstico precoz del melanoma y detectar cambios en nevus preexistentes o nuevas lesiones. En este artículo, presentamos una revisión de los principales factores de riesgo de desarrollar melanoma maligno y las técnicas de detección temprana de melanoma en pacientes de alto riesgo.

Palabras clave: melanoma maligno, nevus melanocítico, factores de riesgo, detección temprana, screening, dermatoscopia.

\begin{abstract}
Malignant melanoma is a type of skin cancer that is characterized by its aggressiveness and treatment resistance. The main risk factors that are associated with development of malignant melanoma are sun exposure and other sources of ultraviolet radiation, the phenotype (hair, eyes and fair skin), the presence of family history of melanoma and dysplastic nevus syndrome. The increased incidence of malignant melanoma in recent decades has created a public health problem and has promoted the development of techniques for early detection of melanoma. Although definitive diagnosis is histological, these non-invasive techniques can help us get an early diagnosis of melanoma and detect changes in existing moles or new lesions. This article presents a review of major risk factors for developing malignant melanoma and early detection techniques of melanoma in high risk patients.
\end{abstract}

Keywords: malignant melanoma, melanocytic nevi, risk factors, early detection, screening, dermoscopy. 


\section{INTRODUCCIÓN}

La incidencia de cáncer cutáneo tipo melanoma está en aumento. En las últimas décadas, se ha duplicado su incidencia y aumenta progresivamente más rápido que cualquier otro cáncer. El melanoma suele afectar a personas jóvenes y de edad media, a diferencia de otros tumores, que son más prevalentes en personas de edad avanzada. Se han desarrollado numerosos tratamientos, pero aun así, los pacientes diagnosticados en etapas avanzadas presentan un mal pronóstico. Por ello, nuestro principal objetivo es el diagnóstico precoz, teniendo en cuenta los factores de riesgo más importantes de melanoma y con un método de screening eficaz.

\section{EPIDEMIOLOGÍA}

La tasa de incidencia estimada del melanoma es de 7,9 por 100.000 personas-año en los países desarrollados. Esta tasa varía según la región del mundo, siendo la más elevada en Australia y Nueva Zelanda, con una tasa media de 33,6 por 100.000 personas-año, seguida de Norteamérica, con una tasa media de 14 por 100.000 personasaño, norte de Europa, con una tasa media de 9,2 por 100.000 personas-año, Europa occidental, con una tasa media de 8,8 por 100.000 personasaño, la zona sur de Europa 5,8, Sudáfrica 4,8, Europa centro-oriental 3,6, Sudamérica 2,4; en Asia, la tasa es menor de 0,5 por 100.000 personas-año ${ }^{1}$.

La tasa de mortalidad aumenta con la tasa de incidencia, pero la variación de la mortalidad entre las distintas regiones del mundo es menor que la variación de la incidencia, con una tasa de mortalidad de 0,1 a 4,0 por 100.000 personas-año².

El melanoma representa el 1,4\% de los cánceres en varones y el 1,6\% de los cánceres en mujeres en todo el mundo, sin tener en cuenta el cáncer cutáneo no melanoma. En países desarrollados, es mayor, casi del doble (2,4 y 2,8\% respectivamente) $)^{3}$.

\section{FACTORES DE RIESGO}

\section{Exposición a radiación ultravioleta}

Es el factor de riesgo medioambiental más importante, sobre todo la exposición solar aguda, intensa e intermitente, principalmente de carácter recreativo, que se valora mediante el antecedente de quemaduras solares ${ }^{4,5}$. Este dato se apoya en la mayor incidencia de melanoma maligno en poblaciones blancas que viven en latitudes bajas. Las personas con historia de quemaduras solares (más de tres quemaduras antes de los 20 años) presentan el doble de riesgo de padecer melanoma maligno que las personas sin este antecedente y es mayor a mayor frecuencia y severidad de las quemaduras ${ }^{6}$. Además, en las zonas con menor capa de ozono, la incidencia de melanoma aumenta, como ocurre en Australia ${ }^{7}$. El riesgo de melanoma se ha asociado con otras características de la piel expuesta a la radiación ultravioleta, como la presencia de otros cánceres cutáneos no melanoma, elastosis solar, lentigos solares y queratosis actínicas, con un riesgo relativo (RR) de 1,9 (95\%, intervalo de confianza [IC] $1,6-2,2)^{8}$. Por otra parte, los pacientes con xeroderma pigmentoso, que se caracterizan por presentar hipersensibilidad a la radiación ultravioleta, presentan mayor riesgo de padecer melanoma maligno en la piel fotoexpuesta ${ }^{9}$ (fig. 1). Otras fuentes de radiación ultravioleta, como las camas de bronceado, las lámparas solares y la terapia PUVA para los pacientes psoriásicos, se han asociado con un aumento del riesgo de melanoma maligno ${ }^{10}$. El empleo de protectores solares lleva a aumentar el tiempo de exposición solar intencionada y puede incrementar la cantidad total de radiación ultravioleta recibida ${ }^{11}$.

\section{Factores hormonales}

Se ha emitido la hipótesis de que los estrógenos y los progestágenos pueden aumentar el riesgo de melanoma mediante la estimulación de la melanogénesis. Se han realizado estudios que demuestran una relación entre los factores reproductivos (edad de menarquia y menopausia, número de 


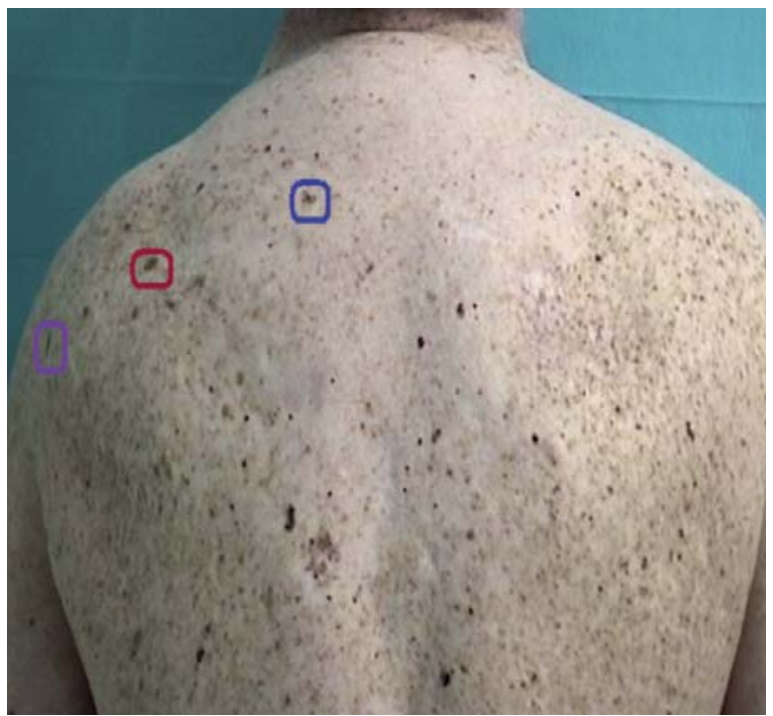

Figura 1. Paciente diagnosticado de xeroderma pigmentoso. Los círculos representan tres lesiones sospechosas.

recién nacidos vivos, etc.) y el riesgo de melanoma, de manera que presentan un menor riesgo las mujeres primíparas jóvenes (menores de 20 años) y las multíparas, por lo que las hormonas endógenas desempeñarían un papel como factor de riesgo. No se ha encontrado aumento del riesgo de melanoma con el uso de anticonceptivos orales o terapia de sustitución hormonal ${ }^{12}$.

\section{Número y tipo de nevus melanocíticos}

Un 25\% de los casos de melanoma aparecen sobre un nevus melanocítico previo ${ }^{13}$. El riesgo de melanoma aumenta con el mayor número de nevus adquiridos, es 1,5 veces mayor en personas con un número de nevus de 11 a 25, comparado con las personas con menos de 10 nevus, y se duplica con cada aumento de 25 nevus. El tamaño de los nevus melanocíticos también se correlaciona con un aumento del riesgo de melanoma (mayores de $5 \mathrm{~mm}$ ), así como la presencia de nevus displásicos. Existen varios datos que apoyan la idea de que el nevus displásico es un marcador de incremento de riesgo de melanoma. Entre un 29 y un $49 \%$ de los melanomas no familiares se dan en pacientes con presencia de nevus displásicos. En personas con historia familiar o personal de melanoma, la presencia de nevus displásico es un marcador de incremento de riesgo de melanoma maligno muy significativo ${ }^{14}$. En pacientes con síndrome del nevus displásico familiar, el riesgo de melanoma se multiplica por 100 y el $50 \%$ de ellos desarrollarán algún melanoma antes de los 50 años. Los nevus displásicos constituyen un factor de riesgo de desarrollar melanomas primarios múltiples.

\section{Edad y sexo}

Son dos factores a tener en cuenta, puesto que los varones presentan más riesgo de melanoma que las mujeres y además, a mayor edad, aumenta el riesgo de melanoma. Hay que tener especial cuidado con los nevus de reciente aparición y con los nevus que presentan cambios en pacientes mayores de 50 años, ya que la probabilidad de que sea melanoma es más alta.

\section{Genética}

Un 8 a 12\% de los melanomas se desarrollan en personas que presentan antecedentes familiares de melanoma ${ }^{15}$. Los pacientes con algún familiar de primer grado afecto presentan el doble de riesgo de padecer melanoma que los que no tienen antecedentes familiares. Este riesgo, a su vez, aumenta si se suman otros factores de riesgo. Las alteraciones genéticas más comunes son las mutaciones en los genes $C D K N 2 A$ y $C D K 4$, siendo esta última menos frecuente ${ }^{16}$. Los pacientes con una predisposición genética de base presentan unas características determinadas, entre las que se incluyen la presencia de múltiples melanomas primarios, pacientes menores de 40 años o con antecedentes de nevus displásicos. Estos pacientes suelen presentar melanomas más superficiales y menos invasivos, que se acompañan de un mejor pronóstico.

\section{Fenotipo}

Los pacientes con pelo de color rojo, rubio o castaño, ojos azules o verdes, sensibilidad solar, mayor 
facilidad para quemaduras, incapacidad de bronceado y presencia de efélides presentan un mayor riesgo de padecer melanoma. Se cree que esto puede estar relacionado con variaciones en el receptor de la melanocortina 1 , ya que se ha observado que los pacientes con variaciones en los alelos de este receptor presentan mayor incidencia de melanomas espontáneos y mayor riesgo de desarrollar melanoma si tienen antecedentes familiares $^{14}$.

\section{Inmunosupresión}

Los pacientes inmunodeprimidos (trasplantados en tratamiento con agentes inmunosupresores, enfermedades hematológicas, sida, etc.) presentan mayor riesgo de padecer melanoma que los pacientes inmunocompetentes.

\section{Otros}

Un nivel socioeconómico bajo se relaciona con melanomas diagnosticados en etapas más avanzadas y con una supervivencia menor.

La presencia de uno o dos de los factores de riesgo citados anteriormente se asocia con un aumento del riesgo de desarrollar un melanoma de 2 a 4 veces mayor que en la población general y, cuando concurren tres factores de riesgo o más, el riesgo se multiplica por $20^{17}$ (tabla 1 ).

\section{PREVENCIÓN DEL MELANOMA}

\section{Prevención primaria}

Consiste en reducir los principales factores de riesgo en poblaciones de alto riesgo. El principal factor de riesgo modificable es la exposición solar. Se debe limitar la cantidad de rayos UVA y UVB recibidos mediante el empleo de prendas de ropa fotoprotectoras, sombreros de ala ancha y aplicación de protectores solares frecuentemente; conviene evitar la exposición solar aguda, intensa e intermitente, sobre todo en las horas centrales del día, y reducir la exposición a otras fuentes de radiación ultravioleta, como las fuentes ocupacionales o camas de bronceado. Es primordial tomar medidas de salud pública, educando a la población sobre la protección solar y especialmente a los padres, para que eviten las quemaduras solares en los niños. Además, es importante promover el autoexamen de la piel, mediante la regla del ABCDE del melanoma (asimetría, bordes, color, diámetro, evolución), en especial en los pacientes con antecedentes personales o familiares de melanoma.

\section{Prevención secundaria}

Se basa en el diagnóstico y el tratamiento precoces. Hay que identificar a los pacientes de alto ries-

Tabla 1. Niveles de evidencia para cada factor de riesgo de melanoma maligno.

\begin{tabular}{|c|c|c|c|}
\hline & Evidencia importante & Evidencia débil & No evidencia \\
\hline Ambientales & $\begin{array}{l}\text { Exposición solar } \\
\text { Localizacion geográfica }\end{array}$ & $\begin{array}{l}\text { Camas de bronceado } \\
\text { Lámparas solares } \\
\text { Obesidad } \\
\text { Ocupación industrial }\end{array}$ & \\
\hline Factor huésped & $\begin{array}{l}\text { No de nevus } \\
\text { Nevus displásico } \\
\text { Ha familiar de melanoma } \\
\text { Inmunosupresión } \\
\text { Sensibilidad solar } \\
\text { Ojos verdes y azules } \\
\text { Pelo rojizo }\end{array}$ & $\begin{array}{l}\text { Cáncer no melanoma } \\
\text { Estatus socioeconómico } \\
\text { Pelo castaño } \\
\text { Sexo masculino } \\
\text { Hormonas endógenas } \\
\text { (menarquia, no partos ) }\end{array}$ & Embarazo \\
\hline
\end{tabular}

Modificado de Svetomir N, et al. ${ }^{16}$. 
go y realizar un seguimiento periódico. Este grupo de pacientes estaría representado por los pacientes que padecen xeroderma pigmentoso, inmunodeprimidos, con nevus displásicos, nevus congénitos gigantes, nevus atípicos, más de 50 nevus, historia familiar de melanoma, etc. Además, conviene entrenar a estos pacientes para que se autoexaminen la piel y se sometan a un seguimiento periódico por el médico.

\section{Prevención terciaria}

El principal objetivo es limitar la morbilidad y aumentar la supervivencia de pacientes con enfermedad avanzada.

\section{DETECCIÓN TEMPRANA DEL MELANOMA}

La detección temprana de melanoma es el factor fundamental para aumentar la supervivencia del paciente y disminuir los costes del tratamiento, ya que los pacientes diagnosticados en etapas avanzadas son los que peor responden al tratamiento y mayores costes conllevan.

No existe un consenso en cuanto al mejor método de screening para una detección temprana del melanoma. Esto es especialmente complicado en los pacientes que presentan múltiples nevus melanocíticos o nevus clínicamente atípicos. Los objetivos para una detección temprana del melanoma consisten en identificar a los pacientes de alto riesgo y realizar un seguimiento periódico de los mismos, monitorizar los nevus y realizar biopsias del melanoma precozmente, evitando procedimientos invasivos innecesarios ${ }^{18}$ (fig. 2).

El screening anual de los pacientes que presentan un alto riesgo es coste-efectivo ${ }^{19}$, y varios estudios demuestran que, además, se asocia con una detección de melanomas más superficiales ${ }^{20}$.

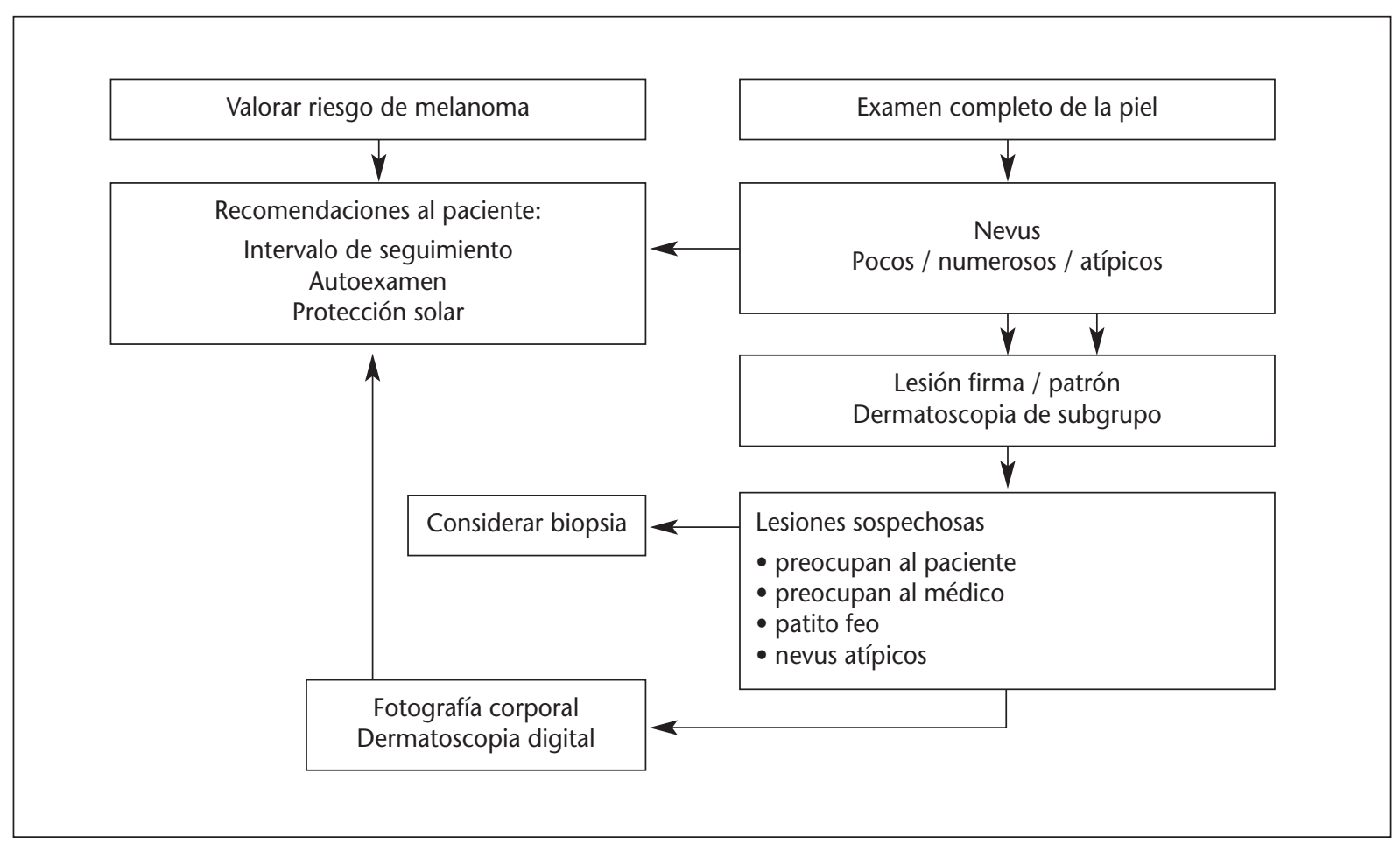

Figura 2. Algoritmo para la evaluación inicial de pacientes con riesgo incrementado para desarrollar melanoma maligno. 


\section{Reconocimiento del melanoma}

La historia de cambios en un nevus preexistente es una alerta que indica posible malignidad. Se ha observado en estudios a corto plazo que la mayoría de los melanomas en crecimiento presentan cambios observables en un período de tiempo que oscila entre 3 y 6 meses $^{21}$. Algunos de estos cambios se han definido como cambios en el color, forma, tamaño, relieve, sangrado, ulceración o prurito y se han asociado a una detección temprana del melanoma.

La aparición de un nuevo nevus puede no ser preocupante, salvo que sea distinto al resto de nevus que presenta el paciente o que aparezca en una persona mayor. El número total de nevus de un paciente presenta un pico en la tercera década de la vida y va disminuyendo en torno a la séptima y octava décadas. Un pequeño porcentaje de estas lesiones será un melanoma, pero la probabilidad aumenta significativamente si estas lesiones se observan en pacientes mayores de 50 años ${ }^{22}$.

\section{Autoexamen de la piel}

Es importante enseñar a los pacientes a realizar autoexámenes de la piel, ya que muchas veces los melanomas son detectados por los pacientes. Estos melanomas suelen ser de mayor espesor que los detectados por los médicos ${ }^{23}$. A su vez, los melanomas detectados por dermatólogos suelen tener menor espesor ( $<0,75 \mathrm{~mm}$ Breslow) que los encontrados por otros profesionales de la salud.

El autoexamen de la piel es especialmente importante para los pacientes que desarrollan melanomas nodulares, ya que, debido al rápido crecimiento de los mismos, pueden aparecer entre una visita y otra al especialista. De esta forma, la responsabilidad de una detección temprana del melanoma se comparte entre el médico y el paciente.

\section{Pacientes con nevus melanocíticos}

El riesgo anual de transformación en melanoma a partir de un nevus preexistente es de $1 / 200.000^{24}$, por lo que surge la duda de si los nevus son precursores de melanoma o sólo marcadores de ries- go. En un $22 \%$ a $50 \%$ de melanomas, se ha observado un origen névico histológicamente ${ }^{13}$, por lo que se podría afirmar que, por lo menos un $50 \%$ de melanomas son de novo. Es por esto por lo que se deben evaluar tanto los nevus de reciente aparición como los cambios en los nevus preexistentes. Al realizar la exploración física, se debe valorar al paciente en conjunto, buscando el patrón mayoritario que presentan los nevus del paciente (nevus con centro oscuro y periferia más clara o viceversa, nevus con borde hiperpigmentado, nevus con patrón de pigmentación concéntrica, nevus con halo, etc.), así como el color, según el fototipo (rosados en pacientes con fototipos I/II, marrón oscuro-negro en fototipos IV-V, etc.). Una vez localizado el patrón más común del paciente, se reduce el número de lesiones sospechosas que requieren realizar una biopsia o un seguimiento más detallado.

Otro dato clínico de ayuda es el conocido signo del «patito feo», que representa una lesión no acorde al resto de nevus del paciente. En pacientes con múltiples nevus de aspecto «normal», el patito feo sería la lesión clínicamente atípica en comparación con el resto, mientras que, en pacientes con múltiples nevus de aspecto atípico, la lesión sospechosa sería la de aspecto más normal (fig. 3).

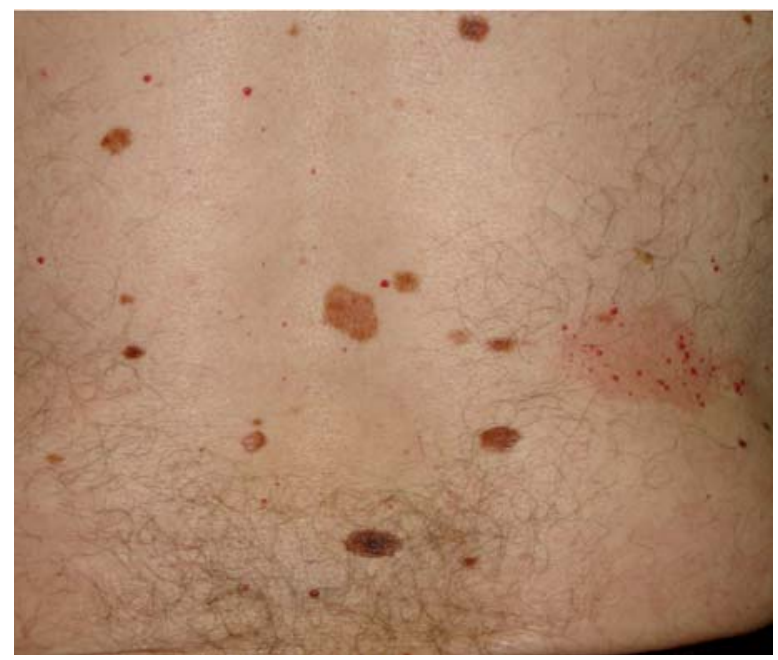

Figura 3. Paciente que presenta múltiples nevus melanocíticos atípicos. 
En el caso de pacientes con pocos o casi ningún nevus, el patito feo sería la lesión solitaria, atípica clínicamente. En un pequeño estudio realizado por Scope et al. ${ }^{25}$, se detectó que el signo del «patito feo" presentaba una sensibilidad del $90 \%$ para la detección del melanoma.

\section{MÉTODOS PARA LA DETECCIÓN DEL MELANOMA}

\section{Examen visual}

Se realiza una historia clínica que refleje los cambios o nuevos nevus que refiere el paciente y posteriormente se examina toda la superficie corporal, enfatizando en las lesiones que han presentado cambios, son nuevas o preocupan al paciente.

\section{Dermatoscopia}

La dermatoscopia, también llamada microscopía de epiluminiscencia, se ha descrito como una herramienta útil para la detección temprana del melanoma y el diagnóstico diferencial de las lesiones pigmentadas de la piel. Es una técnica in vivo que emplea un instrumento magnificador para examinar las lesiones pigmentadas de la piel. Se han descrito múltiples características dermatoscópicas de las lesiones melanocíticas y algoritmos para evaluar la benignidad o malignidad de dichas lesiones, mejorando así la detección temprana del melanoma. La estrategia diagnóstica en dermatoscopia se basa en árboles de decisión, en los que el primer punto consiste en valorar si una lesión es melanocítica o no. Los patrones que apuntan hacia lesiones melanocíticas son: retículo pigmentado, glóbulos, puntos, proyecciones, manchas de pigmento, etc. Después, hay que valorar si la lesión es benigna, sospechosa o maligna, según las características que presente y siguiendo los algoritmos establecidos. Algunos de los criterios dermatoscópicos para la detección de melanoma son los siguientes: retículo pigmentado atípico, velo azul-blanquecino, asimetría, presencia de pseudópodos o estructuras vasculares atípicas, entre otras (fig. 4).

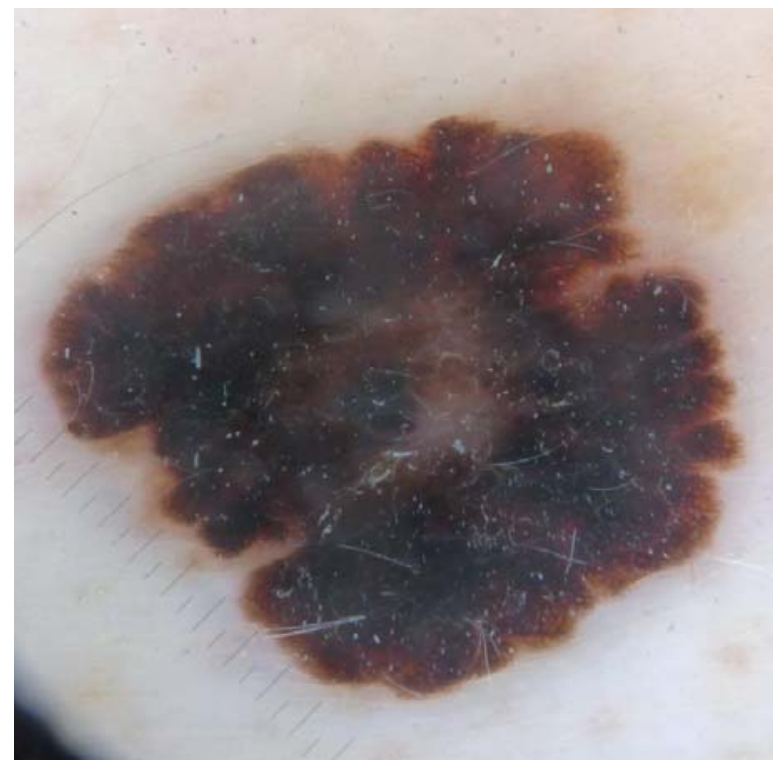

Figura 4. Imagen dermatoscópica de un melanoma de extensión superficial.

Las lesiones sospechosas deben ser biopsiadas, así como las lesiones en las que no se reconoce ningún patrón específico, para descartar un melanoma.

\section{Microscopía confocal}

Permite tomar imágenes de lesiones de la piel en tiempo real, a diferentes profundidades, en planos horizontales, que presentan una correlación dermatoscópica e histológica. En los nevus, se observan nidos de células melanocíticas bien organizados, mientras que en los melanomas se pueden ver melanocitos intraepidérmicos con una desestructuración de la epidermis.

\section{Fotografía total cutánea}

Consiste en realizar fotografías seriadas de toda la superficie cutánea, incluida la piel sin lesiones. Se determinan unas posiciones estándar en la visita inicial para tomar las fotografías y poder realizar comparaciones en cada seguimiento. Este método es muy útil para detectar nuevas lesiones y cambios en el color, la forma o el tamaño de los nevus preexistentes, que pueden sugerir malignidad, si 
bien los cambios muy pequeños pueden no ser detectados con este método.

\section{Dermatoscopia digital asistida por ordenador}

Es un equipo formado por un sistema de lentes magnificadoras acopladas a una cámara óptica conectada a un ordenador, que permite tomar imágenes macro y microscópicas de las lesiones pigmentadas. Las imágenes dermatoscópicas digitales se correlacionan con la imagen macroscópica y a su vez se localizan en un mapa corporal (fig. 5). En cada seguimiento, se refotografían las imágenes dermatoscópicas y, mediante una comparación seriada con las imágenes previas, se valora la presencia de cambios en las lesiones en estudio. Es un método especialmente útil para monitorizar pacientes de alto riesgo que presentan múltiples lesiones melanocíticas. Se obtienen imágenes secuenciales dermatoscópicas en las que se pueden observar características dermatoscópicas que permiten diagnosticar melanomas incipientes que clínicamente carecen de criterios de melanoma.

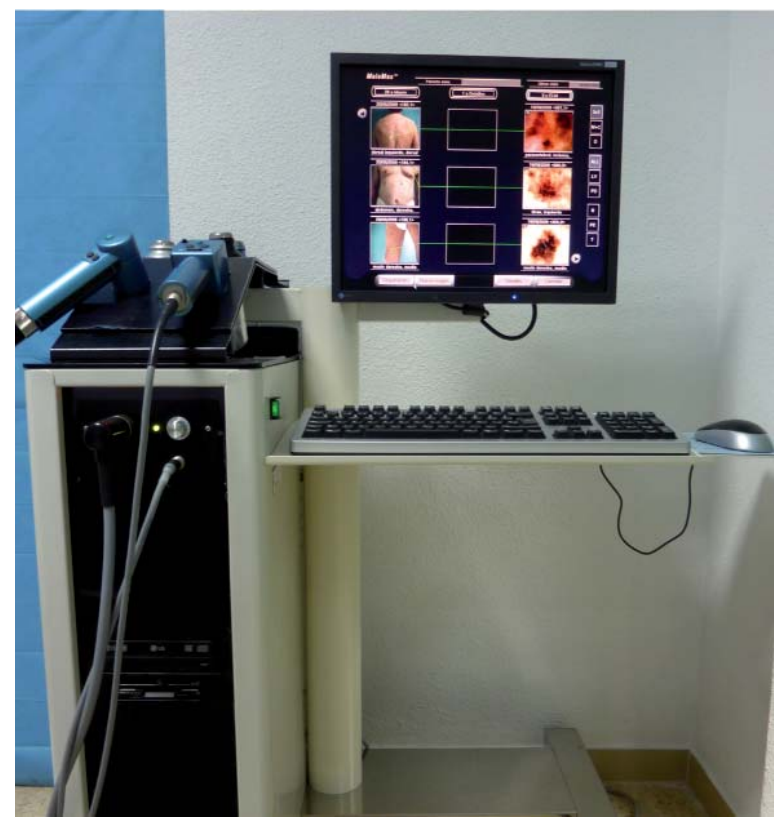

Figura 5. Unidad de dermatoscopia y fotografía digital.

\section{Teledermatoscopia}

Es una nueva técnica en crecimiento. Sirve para realizar una evaluación rápida de lesiones sospechosas, sin que el paciente tenga que esperar mucho tiempo hasta la consulta con el especialista ni desplazarse en busca de un experto. Nueva Zelanda ha sido el primer país en comercializar la teledermatoscopia, debido a la alta demanda de screening de melanoma y a la escasez de dermatólogos. Los pacientes acuden al centro de salud, donde una enfermera o médico entrenados les toman fotografías clínicas y dermatoscópicas de las lesiones sospechosas y les hacen una serie de preguntas sobre su tipo de piel. Estos datos e imágenes se envían electrónicamente a un dermatoscopista experto, quien los valorará y enviará de vuelta un informe con el diagnóstico provisional y las recomendaciones sobre si debe ser extirpada la lesión o no, así como el plan a seguir.

\section{Dermatoscopia digital multiespectral}

Se emplea una luz de longitud de onda variable, que penetra en la piel a distintas profundidades y nos permite obtener imágenes secuenciales que nos informan sobre el contenido de melanina, la distribución del colágeno y la vascularización de las lesiones cutáneas. Estas imágenes se analizan y se comparan con una base de datos de lesiones benignas y melanomas. Éste es un método cuantitativo y, por tanto, más objetivo que la dermatoscopia que, al ser un método cualitativo, admite mayor subjetividad.

\section{Otros}

Imágenes por ultrasonido de alta resolución, tomografía de coherencia óptica, imágenes por resonancia magnética, perfiles moleculares, etc.

\section{CONCLUSIONES}

A pesar de que la incidencia de melanoma continúa aumentando, la mortalidad tiende a estabilizarse, gracias a los programas de prevención del melanoma y a los avances en el diagnóstico pre- 
Burgués Calderón M et al. Factores de riesgo y detección temprana del melanoma maligno en pacientes con nevus melanocíticos

coz. La necesidad de disminuir las muertes debidas a melanoma, mediante la detección temprana del melanoma, ha promovido el desarrollo de nuevas técnicas con una mejor sensibilidad diagnóstica. Entre ellas, las más difundidas en la práctica clínica son la fotografía cutánea total y la dermatoscopia digital. La fotografía cutánea es esencial para detectar nuevas lesiones o la presencia de cambios en lesiones preexistentes, y la dermatoscopia nos ayuda a diferenciar si una lesión melanocítica es benigna o maligna cuando clínicamente no lo apreciamos. Cualquier técnica presenta sus limitaciones, por lo que lo ideal sería combinar varias modalidades para optimizar la detección temprana del melanoma. Se puede presumir que, en un futuro, los sistemas multimodales serán los que proporcionen un diagnóstico más preciso. Mediante los programas de prevención eficaces, la incorporación de autoexámenes de la piel y junto con las múltiples técnicas de detección de melanoma, la mortalidad por melanoma se podría reducir a través del diagnóstico precoz, llegando a conseguir no encontrar pacientes con melanoma invasivo en las visitas de seguimiento.

\section{BIBLIOGRAFÍA}

1. Ferlay J, Bray F, Pisani P, Parkin DM. GLOBOSCAN 2002: Cancer Incidence, Mortality and Prevalence Worldwide. Version 2.0. Lyon: IARC Cancerbase No 5; 2004.

2. Armstrong B. Epidemiology of melanoma and current trends. En: Thompson JF, Morton DL, Kroon BBR, eds. Textbook of melanoma. Londres: Martin Dunitz; 2004.

3. Balch C, Houghton A, Sober A, Soong SI, Atkins MB, Thompson JF. Cutaneous melanoma. 5. ${ }^{\mathrm{a}}$ ed. St Louis: Quality Medical Publishing, Inc.; 2009.

4. Elwood JM, Jopson J. Melanoma and sun exposure: an overview of published studies. Int | Cancer. 1997;73:198-203.

5. Veierød MB, Weiderpass E, Thörn M, Hansson J, Lund E, Armstrong $B$, et al. A prospective study of pigmentation, sun exposure and risk of cutaneous malignant melanoma in women. J Natl Cancer Inst. 2003;95:1530-8.

6. Gandini S, Sera F, Cattaruzza MS, Pasquini P, Picconi O, Boyle $\mathrm{P}$, et al. Meta-analysis of risk factors for cutaneous melanoma II. Sun exposure. Eur J Cancer. 2005;41:45-60.

7. Goldstein AM, Tucker MA. Etoilogy, epidemiology, risk factors and public health issues of melanoma. Curr Opin Oncol. 1993;5:358-363.

8. Gruber SB, Armstrong BK. Cutaneous and ocular melanoma. En: Schottenfeld D, Fraumeni JF, eds. Cancer epidemiology and prevention. Nueva York: Oxford University Press; 2006.
9. Kraemer $\mathrm{KH}$, Lee MM, Andrews AD, Lambert WC. The role of sunlight and DNA repair in melanoma and nonmelanoma skin cancer: the xeroderma pigmentosum paradigm. Arch Dermatol. 1994;130:1018-21.

10. Stern RS. The risk of melanoma in association with long-term exposure to PUVA. J Am Acad Dermatol. 2001;44:755-61.

11. Autier P, Dore JF, Reis AC, Grivegnée A, Ollivaud L, Truchetet $F$, et al. Sunscreen use and intentional exposure to ultraviolet $A$ and $B$ radiation: $a$ double blind randomized trial using personal dosimeters. Br J Cancer. 2000;83:1243-8.

12. Karagas MR, Zens MS, Stukel TA, Swerdlow AJ, Rosso S, Osterlind $A$, et al. Pregnancy history and incidence of melanoma in women: a pooled analysis. Cancer Causes Control. 2006;17:11-9.

13. Bevona C, Goggins W, Quinn T, Fullerton J, Tsao H. Cutaneous melanomas associated with nevi. Arch Dermatol. 2003;139: 1620-4.

14. Markovic SN, Erickson LA, Rao RD, Weenig RH, Pockaj BA, Bardia A, et al. Malignant melanoma in the 21st century, part 1: epidemiology, risk factors, screening, prevention and diagnosis. Mayo Clin Proc. 2007;82(3):364-80.

15. Goldstein AM, Tucker MA. Genetic epidemiology of cutaneous melanoma: a global perspective. Arch Dermatol. 2001;137: 1493-6.

16. Harland M, Taylor CF, Bass S, Churchman M, Randerson-Moor JA, Holland EA, et al. Intronic sequence variants of the CDKN2A gene in melanoma pedigrees. Genes Chromosome Cancer. 2005;43:128-36.

17. Cho E, Rosner BA, Feskanich D, Colditz GA. Risk factors and individual probabilities of melanoma for whites. J Clin Oncol. 2005; 14:1241-4

18. Gadeliya A, Grossman D. Strategies for early melanoma detection: Approaches to the patient with nevi. J Am Acad Dermatol. 2009;60:719-35

19. Freedberg KA, Geller AC, Miller DR, Lew RA, Koh HK. Screening for malignant melanoma: a cost-effectiveness analysis. J Am Acad Dermatol. 1999;41:738-45.

20. Carli P, De Giorgi V, Palli D, Maurichi A, Mulas P, Orlandi C, et al. Dermatologist detection and skin self-examination are associated with thinner melanomas: results from a survey of the Italian Multidisciplinary Group on Melanoma. Arch Dermatol. 2003;139:607-12.

21. Altamura D, Avramidis M, Menzies SW. Assesment of the optimal interval for and sensitivity of short-term sequential digital dermoscopy monitoring for the diagnosis of melanoma. Arch Dermatol. 2008;144:502-6.

22. Banky JP, Kelly JW, English DR, Yeatman JM, Dowling JP. Incidence of new and changed nevi and melanomas detected using baseline images and dermoscopy in patients at high risk for melanoma. Arch Dermatol. 2005;141:998-1006.

23. Epstein DS, Lange JR, Gruber SB, Mofid M, Koch SE. Is physician detection associated with thinner melanomas? JAMA. 1999;281:640-3

24. Tsao H, Bevona C, Goggins W, Quinn T. The transformation rate of moles (melanocytic nevi) into cutaneous melanoma: a population-based estimate. Arch Dermatol. 2003;282-8.

25. Scope A, Duzsa SW, Halpem AC, Rabinovitz H, Braun RP, Zalaudek I, et al. The «ugly duckling» sign: agreement between observers. Arch Dermatol. 2008;144:58-64. 